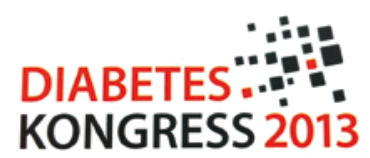

\section{Weitere seltene Diabetesformen}

- Diabetes bei Zystischer Fibrose:

Mit zunehmender Lebenserwartung von Patienten mit Mukoviszidose steigt auch der Anteil von Ko-Morbiditäten. Dazu gehört auch der Diabetes mellitus. Das Alter der Patienten bei Manifestation ist im Mittel höher als bei einem Typ-1-Diabetes, aber niedriger als bei Typ 2- Diabetes. Auch ist der BMI niedriger als bei Typ-2- Diabetikern.

\section{- Diabetes nach Nierentransplantation:}

Die Neumanifestation eines Diabetes mellitus nach einer Nierentransplantation ist keine Rarität. Ursächlich werden mit der Transplantation einhergehende Ischämie-Reperfusionsmechanismen diskutiert, die prädisponierend sind für eine Insulinresistenz. Darüber hinaus können Immunsuppressiva wie Calcineurininhibitoren und Steroide die Insulinausschüttung hemmen und die Insulinresistenz verstärken. Bei vielen betroffenen Patienten bestand bereits vor der Transplantation eine Insulinresistenz. Ein isolierter Sekretionsdefekt in Folge einer toxischen Schädigung der Betazelle durch Immunsuppressiva konnte innerhalb der ersten 12 Monate nach der Transplantation nicht nachgewiesen werden.

\section{- DPP-4-Genvariante:}

Die Dipeptidyl-Peptidase 4 spaltet und inaktiviert die insulinotropen Inkretine wie GLP-1. Der klinische Alltag zeigt eine beachtliche interindividuelle Variation in der metabolischen Antwort auf DPP-4-Inhibitoren als Ausdruck einer DPP-4-Genvariation. Eine besondere Genvariante von DDP-4 interagiert mit dem Körperfett und vermindert die durch Glukose stimulierten GLP-1-Spiegel, die Insulinsekretion und die Glukosetoleranz.

\section{- Prader-Willi-Syndrom:}

Das Prader-Willi-Syndrom ist eine genetische Erkrankung, die durch Adipositas, Intelligenzminderung, Impulskontrollstörung und Minderwuchs gekennzeichnet ist. Folgeerkrankungen wie ein Diabetes mellitus Typ 2 und Herz-Kreislauf-Erkrankungen sind häufig. Therapeutisch empfehlenswert sind lebensdiätetische Maßnahmen wie strikte Diät und sportliche Aktivitäten.

Dr. med. Peter Stiefelhagen

und zu einer Verschlechterung der Glukosetoleranz bis hin zum Diabetes mellitus. „Doch nach einer Pankreaskopfresektion ist die Glukosetoleranz aufgrund einer verzögerten Magenentleerung sogar verbessert", so Meier. Auch sei das Diabetesrisiko insgesamt gering, d.h. nach 3,5 Jahren entwickelt nur jeder vierte operierte Patient einen Diabetes mellitus, wobei ein erhöhtes Körpergewicht und eine bereits vorbestehende Störung des Kohlenhydratstoffwechsels die Manifestation begünstigen.

\section{Typ-1-Diabetes als Teil eines polyglandulären}

\section{Autoimmunsyndroms}

Der Typ-1-Diabetes ist nicht selten mit anderen Autoimmunerkrankungen assoziiert. Dazu gehören Immunthyreopathien wie der Morbus Basedow oder die Hashimoto-Thyreoiditis, die Sprue, die perniziöse Anämie und die Autoimmunadrenalitis mit konsekutiver Unterfunktion der Nebennieren. „Der Nachweis solcher begleitender Autoimmunerkrankungen ist wichtig, da diese Einfluss auf den Glukosestoffwechsel nehmen und eine Anpassung der Insulindosierung erforderlich machen“, sagte Frau Prof. Wilgard Hunger-Battefeld von der Me- dizinischen Universitätsklinik in Jena. So führe eine Hyperthyreose bei einem Morbus Basedow zu einer zunehmenden Insulinresistenz und $\mathrm{zu}$ einer vermehrten Glukagonfreisetzung mit konsekutivem Blutzuckeranstieg und der daraus resultierenden Notwendigkeit der Insulindosis-Steigerung. Umgekehrt erhöht eine Hypothyreose die Insulinsensitivität, sodass evtl. die Insulindosis verringert werden muss. Bei einem Morbus Addison fehlen antiinsulinäre Hormone. Es besteht die Gefahr einer schweren Hypoglykämie mit Bewusstlosigkeit. „Und bei Patienten mit einer perniziösen Anämie wird die durch Vitamin $B_{12}$-Mangel verursachte neurologische Symptomatik öfter als diabetische Polypneuropathie fehlinterpretiert", so Hunger-Battefeld.

Beim polyglandulären Autoimmunsyndrom (PAS) werden zwei Typen, eine juvenile und eine adulte Form unterschieden. Beim Typ 1, der auch Blizzard-Syndrom genannt wird, handelt es sich um eine autosomal-rezessiv vererbte Endokrinopathie als Folge einer monogenetischen Störung im Bereich des Autoimmune Regulator Gens (AIRE) auf dem Chromosom 21q22.3. Die AIRE-Mutation verhindert die Eliminierung von potenziell autoreaktiven T-Zellen, was in bestimmten Geweben zur Prädisposition für eine Autoimmunreaktion führt. Der PAS Typ 2 tritt familiär gehäuft auf. Hierbei handelt es sich um eine komplexe polygenetische Störung mit hoher Variabilität, die autosomal dominant vererbt wird.

\section{Wann und wie sollten Typ-1-Diabetiker auf} Autoimmunendokrinopathien hin gescreent werden?

Entsprechende Untersuchungen haben gezeigt, dass über 50\% der Typ-1-Diabetiker mindestens einen weiteren pathologischen Autoimmunantikörpertiter aufweisen, der jedoch noch keinen sicheren Rückschluss auf eine klinisch symptomatische Erkrankung eines anderen Organs zulässt. „Deshalb ist ein generelles Autoantikörper-Screening für die klinische Betreuung dieser Patienten meist nicht hilfreich", so Hunger-Battefeld. Doch jeder dritte Typ-1-Diabetiker entwickelt eine weitere therapiepflichtige Autoimmunendokrinopathie, wobei jährlich mit einem Prävalenzanstieg von 3,6\% gerechnet werden muss. Deshalb sollte bei entsprechender Symptomatik immer an ein polyglanduläres Autoimmunsyndrom gedacht und eine weitere Abklärung veranlasst werden. Dazu gehört die Kontrolle der Nebennierenrindenfunktion, des Blutbildes, des Kalziumstoffwechsels und auch der gonadalen Funktion.

Die häufigste Zweiterkrankung bei einem Typ-1-Diabetiker ist die Hashimoto-Thyreoiditis oder ein Morbus Basedow. Risikomarker sind ein höheres Alter, eine längere Diabetesdauer und weibliches Geschlecht. Angesichts der Häufigkeit der Thyreopathie kann ein entsprechendes Autoantikörper-Screening, speziell die Bestimmung von Anti-TPO und TAK sinnvoll sein. „Im Rahmen der Betreuung von Typ-1-Diabetikern sollte besonders bei Patienten mit pathologisch erhöhten Schilddrüsenautoantikörpern eine jährliche TSH-Kontrolle erfolgen“, so die Empfehlung von Hunger-Battefeld.

Dr. med. Peter Stiefelhagen

Quelle: Symposium „Sonderformen des Diabetes“ am 9.5.2013 beim Diabeteskongress in Leipzig. 